\title{
Gradiente residual em operação de valva aórtica
}

\author{
Zildomar DEUCHER JUNIOR* ${ }^{*}$ Eduardo Sérgio BASTOS* , José Leôncio de Andrade FEITOSA*, \\ Rubens GIAMBRONI FILHO*, José Augusto Pereira de AZEVEDO*, Mauro Paes Leme de SÁ*, \\ Álvaro Barde BEZERRA*, Antônio de Pádua JAZBIK*, Henrique MURAD*
}

RBCCV 44205-495

Deucher Júnior Z, Bastos E S, Feitosa J L A, Giambroni Filho R, Azevedo J A P, Sá M P L, Bezerra A B, Jazbik A P, Murad H - Gradiente residual em operação de valva aórtica. Rev Bras Cir Cardiovasc $2000 ; 15(2): 136-42$.

RESUMO: Objetivo: Analisar o gradiente pressórico residual após cirurgia de troca valvar aórtica realizada no Hospital Universitário Clementino Fraga Filho - UFRJ.

Casuística e Métodos: Estudo retrospectivo envolvendo pacientes submetidos a operação de troca valvar aórtica isolada em nosso Serviço, no período compreendido entre janeiro de 1988 e dezembro de 1998. Foram incluídos apenas os pacientes com diagnóstico de estenose aórtica isolada, que receberam prótese de tamanho 23 ou menor e que tenham sido submetidos a estudo ecocardiográfico pré e pósoperatório em nossa Instituição. Atenderam aos critérios 44 pacientes. Predominou o sexo masculino com $63,6 \%$ dos casos, a média de idade de 53,9 anos e superfície corpórea média de 1,67 $\mathrm{m}^{2}$. A prótese utilizada foi biológica em 25 pacientes e mecânica em 19, sendo 8 de monofolheto e 11 de duplo folheto. Antes da operação, 12 pacientes encontravam-se em classe funcional II, 28 em classe III e 4 em classe IV. E o gradiente era em média $95,8 \mathrm{mmHg}$.

Resultados: Seis meses após a operação, 35 (79,5\%) pacientes estavam em classe I e os demais $(20,5 \%)$ em classe II. A variação de gradiente foi em média $62,9 \mathrm{mmHg}$, chegando-se ao gradiente final de $32,9 \mathrm{mmHg}$ em média. A variação de gradiente não foi influenciada por sexo, idade, superfície corpórea ou tamanho da prótese. Foi, entretanto, influenciada diretamente pelo gradiente pré-operatório. $\mathrm{O}$ gradiente pós-operatório foi em média $24,3 \mathrm{mmHg}$ para próteses de duplo folheto, $33,7 \mathrm{mmHg}$ para biopróteses e 42,3 $\mathrm{mmHg}$ para as próteses de monofolheto.

Conclusão: A variação de gradiente foi diretamente proporcional ao gradiente pré-operatório. 0 melhor resultado em termos de gradiente residual foi das próteses de duplo folheto, seguidas pelas biopróteses e, finalmente, pelas próteses de monofolheto. A utilização de próteses de tamanho menor que o recomendado não determinou a ocorrência de gradientes residuais mais elevados.

DESCRITORES: Valva aórtica, cirurgia. Valva aórtica, fisiologia. Estenose da valva aórtica, cirurgia. implante de prótese de valva.

\section{INTRODUÇÃO}

O tratamento cirúrgico da doença valvar aórtica sofreu grande evolução, desde o trabalho pioneiro do Dr. Hufnagel, que implantou uma prótese na aorta descendente para correção de insuficiência aórtica. Em 1960, o Dr. Harken implantou, pela primeira vez, uma prótese valvar aórtica em posição subcoronária (1). Durante as décadas que se segui-

Trabalho realizado no Hospital Universitário Clementino Fraga Filho - UFRJ. Rio de Janeiro, RJ, Brasil. Apresentado ao 27ํㅡㄹ Congresso Nacional de Cirurgia Cardíaca. Rio de Janeiro, 23 a 25 de março, 2000. *Do Hospital Universitário Clementino Fraga Filho. 
Deucher Júnior Z, Bastos E S, Feitosa J L A, Giambroni Filho R, Azevedo J A P, Sá M P L, Bezerra A B, Jazbik A P, Murad H - Gradiente residual em operação de valva aórtica. Rev Bras Cir Cardiovasc 2000; 15(2): 136-42.

ram, numerosos modelos de próteses foram desenvolvidos por diversos pesquisadores à procura de um desenho que conciliasse, entre outras qualidades, facilidade de implante, bom desempenho hemodinâmico e durabilidade. O passar do tempo demonstrou que alcançar este objetivo não é tarefa fácil e, na verdade, ainda não chegamos ao substituto valvar ideal.

Apesar do desenvolvimento de técnicas de plastia valvar aórtica, a maioria dos pacientes portadores de doença desta valva que chega à operação necessita de substituição valvar. Embora existam outras opções, tais como uso de homoenxerto aórtico e autoenxerto pulmonar, a maior parte dos pacientes recebem próteses convencionais, biológicas ou mecânicas. Dentre as próteses biológicas existem aquelas sustentadas por um anel, e as que prescindem do uso do anel ditas stentless. Já as próteses mecânicas são invariavelmente montadas em um anel, diferenciando-se pelo número e desenho dos folhetos.

O gradiente residual transprotético é um dos fatores determinantes do resultado final dos pacientes, mormente naqueles que apresentam anel reduzido por hipotrofia ou calcificação.

O objetivo deste trabalho é analisar o gradiente pressórico residual após operação de troca valvar aórtica realizada no Hospital Universitário Clementino Fraga Filho.

\section{CASUÍSTICA E MÉTODOS}

Este trabalho é baseado em estudo retrospectivo de análise de prontuários dos pacientes submetidos a troca valvar aórtica isolada no Serviço de Cirurgia Cardiovascular do Hospital Universitário Clementino Fraga Filho da Universidade Federal do Rio de Janeiro, durante o período compreendido entre janeiro e dezembro de 1998.

Foram selecionados apenas os pacientes com diagnóstico de estenose aórtica isolada, submetidos a estudo ecocardiográfico pré e pós-operatório em nossa Instituição, que tenham recebido implan- te de prótese tamanho 23 ou menor.

Atenderam aos critérios de inclusão no estudo 44 pacientes. Dentre estes $28(63,6 \%)$ eram do sexo masculino. A idade variou entre 16 e 72 anos $(\mathrm{m}=$ $53,9 \pm 13,7$ anos). A superfície corpórea variou de 1,30 a $2,00 \mathrm{~m}^{2}\left(\mathrm{~m}=1,67 \mathrm{~m}^{2}\right)$. O gradiente préoperatório variou de 52 a $173 \mathrm{mmHg}$, com média de $95,8 \mathrm{mmHg}$ (Tabela 1).

A classe funcional pré-operatória segundo a NYHA estava assim distribuída: 12 pacientes em classe funcional II, 28 pacientes em classe III, e 4 pacientes em classe funcional IV.

Foram implantadas 25 biopróteses e 19 próteses mecânicas, dentre estas 8 de monofolheto e 11 de duplo folheto. Quanto ao tamanho da prótese, apenas 1 paciente recebeu prótese 19, 24 pacientes receberam prótese 21 e 19 receberam prótese 23 . Não foram utilizadas próteses stentless.

Os dados procurados foram a variação de gradiente e o gradiente residual final, e a relação destes com idade, sexo, superfície corpórea, tamanho e tipo de prótese.

Os dados obtidos foram analisados através de programa estatístico EPI INFO Versão 5.01 (Epidemiology Program Office, Centers for Disease Control, Atlanta, Georgia).

\section{RESULTADOS}

A variação de gradiente (delta $G$ ) não foi influenciada pela idade ou superfície corpórea. Foi um pouco maior no sexo feminino com média de $70,8 \mathrm{mmHg}$, que no masculino com média de $58,4 \mathrm{mmHg}$, entretanto, sem significância estatística $(p=0,299)$. Quando agrupadas as próteses por tamanho, não observamos diferença significativa entre próteses de tamanho 21 e 23 ( $p=0,769)$ (Gráfico 1).

A variação de gradiente foi influenciada diretamente pelo gradiente pré-operatório (Gráfico 2). A variação foi maior nas próteses mecânicas de duplo folheto com média $71,3 \mathrm{mmHg}$, seguidas das biopróteses com média de $66,7 \mathrm{mmHg}$ e, finalmen-

TABELA 1

ESTATÍSTICA DESCRITIVA DE ALGUMAS VARIÁVEIS NUMÉRICAS

\begin{tabular}{lccccc}
\hline VARIÁVEIS & MÉDIA & DP & MÍNIMO & MEDIANA & MÁXIMO \\
\hline Superfície corpórea (m $\left.{ }^{2}\right)$ & 1,67 & 0,18 & 1,3 & 1,70 & 2,0 \\
Gradiente pré (mmHg) & 95,8 & 29,6 & 52,0 & 90,5 & 173,0 \\
Gradiente pós (mmHg) & 32,9 & 13,0 & 12,0 & 30,0 & 70,0 \\
$\Delta$ Gradiente (mmHg) & 62,9 & 30,3 & 7,0 & 59,0 & 129,0 \\
Follow-up (meses) & 63,3 & 39,6 & 3,0 & 55,0 & 129,0 \\
\hline
\end{tabular}


Deucher Júnior Z, Bastos E S, Feitosa J L A, Giambroni Filho R, Azevedo J A P, Sá M P L, Bezerra A B, Jazbik A P, Murad H - Gradiente residual em operação de valva aórtica. Rev Bras Cir Cardiovasc 2000; 15(2): 136-42.

GRÁFICO 1

VARIAÇÃODOGRADIENTE SEGUNDO O TAMANHODA PRÓTESE

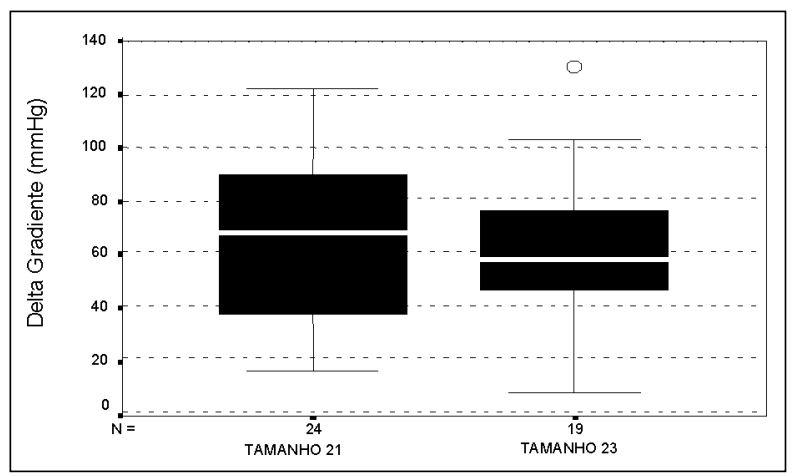

GRÁFICO2

VARIACSÃODOGRADIENTE SEGUNDOGRADIENTE PRÉ-OPERATÓRIO

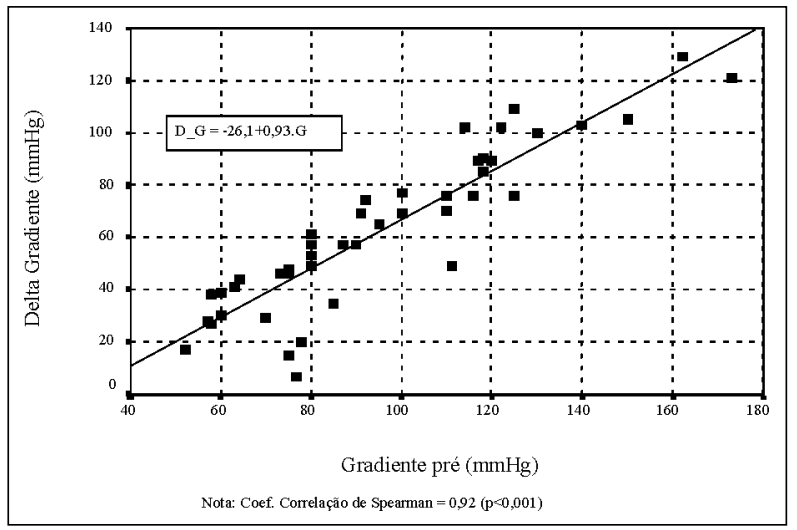

te, as mecânicas de monofolheto com média de $39,6 \mathrm{mmHg}$ (Gráfico 3). Este dado, entretanto, não pode ser sobrevalorizado, pois a análise dos gradientes pré-operatórios revela que estes eram mais baixos nos pacientes que receberam próteses mo-

GRÁFICO 3

VARIAÇÃODOGRADIENTE SEGUNDO TIPODEPRÓTESE

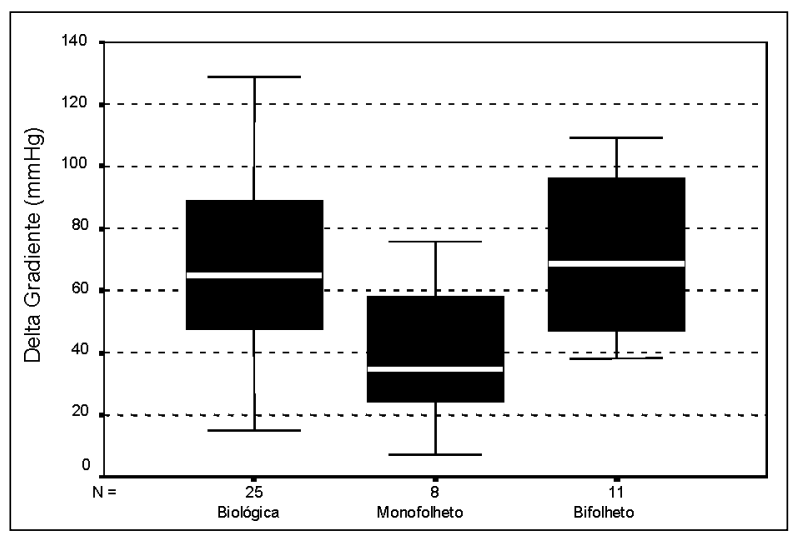

nofolheto com média de $81,9 \mathrm{mmHg}$, que naqueles que receberam próteses de duplo folheto que apresentavam média de $95,5 \mathrm{mmHg}$, ou biopróteses com 100,4 mmHg (Gráfico 4).

Quando passamos a analisar o gradiente final, observamos que este não sofreu influência de fatores tais como: sexo, idade ou superfície corpórea. É interessante notar que não houve também influência do tamanho da prótese, uma vez que o gradiente final médio das próteses tamanho 21, agrupados todos os modelos, foi $35,1 \mathrm{mmHg}$, ao passo que o das próteses tamanho 23 foi $30,9 \mathrm{mmHg}(p=0,159)$ (Gráfico 5). Da mesma forma, não houve correlação direta entre o índice valvar (tamanho da prótese/ superfície corpórea) e o gradiente final observado (Gráfico 6).

A análise do gradiente final no pós-operatório foi influenciada pelo tipo de prótese utilizado. Observamos nítida superioridade dos resultados obtidos com o uso de próteses mecânicas de duplo folheto, cujo gradiente final foi em média $24,3 \mathrm{mmHg}$, quando comparadas as biopróteses com média $33,7 \mathrm{mmHg}$

GRÁFICO 4

GRADIENTE PRÉ-OPERATÓRIO SEGUNDO TIPODEPRÓTESE

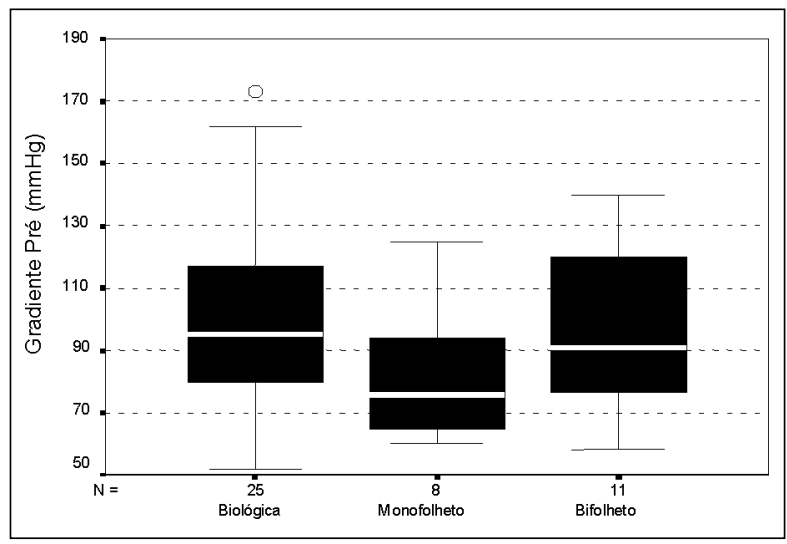

GRÁFICO 5

GRADIENTE FINAL SEGUNDOO TAMANHO DA PRÓTESE

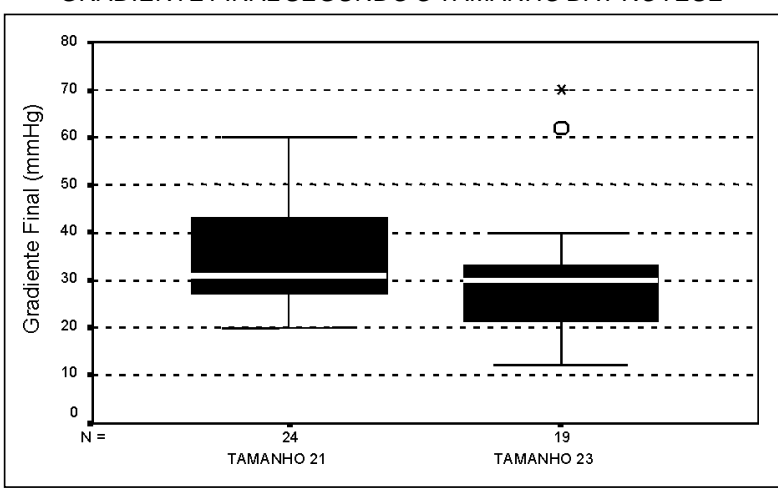


Deucher Júnior Z, Bastos E S, Feitosa J L A, Giambroni Filho R, Azevedo J A P, Sá M P L, Bezerra A B, Jazbik A P, Murad H - Gradiente residual em operação de valva aórtica. Rev Bras Cir Cardiovasc 2000; 15(2): 136-42.

e próteses mecânicas de monofolheto com média 42,3 mmHg (Gráfico 7).

$\mathrm{Na}$ avaliação pós-operatória, realizada seis meses após as operações, $35(79,5 \%)$ pacientes encontravamse em classe I e $9(20,5 \%)$ em classe II.

\section{COMENTÁRIOS}

Pacientes portadores de estenose aórtica beneficiam-se da operação de troca valvar aórtica, pois esta possibilita uma grande redução da pós-carga do ventrículo esquerdo, fazendo-se acompanhar de melhora clínica acentuada. Esta melhora está geralmente associada a uma importante redução da hipertrofia muscular do ventrículo esquerdo ${ }^{(2)}$. A utilização de próteses valvares com bom desempenho hemodinâmico e pequenos gradientes transprotéticos é de fundamental importância, uma vez que, a presença de gradientes pressóricos residuais elevados é causa de não regressão da hipertrofia ventricular esquerda, que, por sua vez, está relacionada com maior morbidade, manifestada na forma de arrit-

GRÁFICO 6

GRADIENTE FINAL SEGUNDO RELAÇÃO TAMANHO DE PRÓTESE/ SUPERFÍCIE CORPÓREA

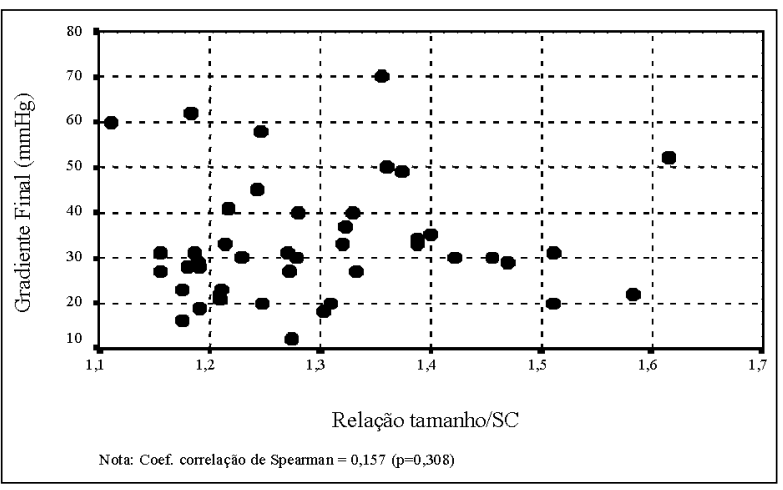

GRÁFICO 7

GRADIENTE PÓS-OPERATÓRIO SEGUNDO TIPODEPRÓTESE

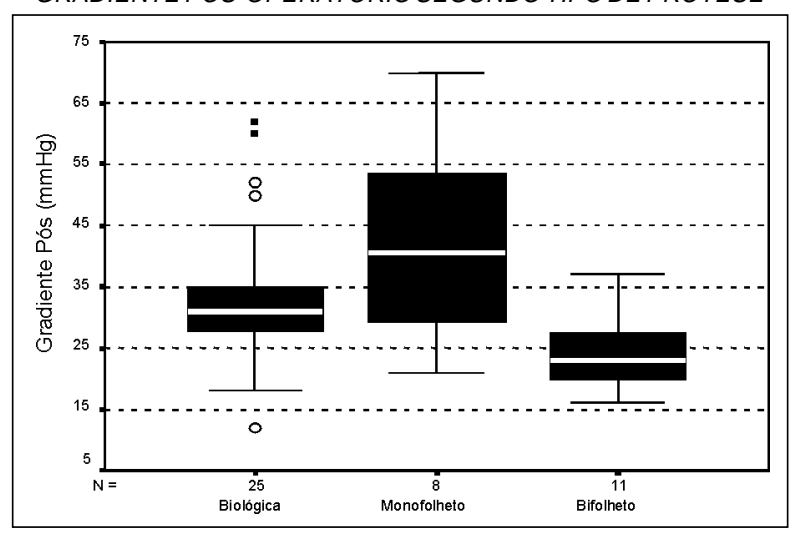

mias e insuficiência cardíaca, e mortalidade tardia, notadamente a morte súbita (3-5).

Grande é a discussão em torno do tamanho apropriado de próteses valvares, pois a utilização de próteses pequenas é considerada responsável pela permanência de gradientes residuais no pósoperatório, com seus efeitos deletérios (2, 6-8).

O presente estudo foi concebido com a intenção de conhecer a real situação no que tange a gradientes residuais em nossa experiência.

A utilização do ecocardiografia com Doppler como método de avaliação de gradientes transprotéticos está bem estabelecida, contando ainda com a vantagem adicional de não ser invasivo, sendo, por isso mesmo, o método mais empregado na prática clínica $(6,9)$.

A população pequena deve-se à exclusão de pacientes com doença cardíaca associada, valvar ou não, bem como daqueles com diagnóstico de insuficiência aórtica ou endocardite infecciosa tratados cirurgicamente em nosso Serviço, e à dificuldade de seguimento e reavaliação dos pacientes vindos de outros estados.

A análise da variação do gradiente demonstrou não estar a mesma relacionada a idade, sexo ou superfície corpórea do paciente e nem mesmo com o tamanho da prótese implantada. Apesar de ter sido observada maior variação de gradiente após utilização de prótese de duplo folheto ou de biopróteses que, quando do uso de próteses de monofoIheto, a constatação de que, estas últimas apresentavam a média mais baixa de gradiente pré-operatório, explica o achado uma vez que o gradiente pré-operatório influencia diretamente a variação do gradiente.

O gradiente residual final foi menor nas próteses mecânicas de duplo folheto com média de $24,3 \mathrm{mmHg}$, seguido pelas biopróteses ( $\mathrm{m}=33,7 \mathrm{mmHg}$ ) e mecânicas de monofolheto $(\mathrm{m}=42,3 \mathrm{mmHg})$, o que está de acordo com a literatura $(6,8,10-13)$.

A análise do gradiente final demonstrou que não houve influência direta do sexo, idade e superfície corpórea isoladamente. Surpreende, até certo ponto, o achado de não haver diferença estatística entre próteses tamanho 21 e 23, o que pode, entretanto, ser creditado à seleção de prótese de tamanho adequado para cada paciente da amostra. Entretanto, não houve relação direta entre o gradiente final e o índice valvar (tamanho de prótese/superfície corpórea) (Gráfico 6).

Aceita-se geralmente uma superfície corpórea menor que $1,7 \mathrm{~m}^{2}$ como ideal para uso de próteses pequenas ${ }^{(6)}$. SOMMERS \& DAVID ${ }^{(14)}$ sugerem tamanho 21 para pacientes com até $1,5 \mathrm{~m}^{2}$, tamanho 
Deucher Júnior Z, Bastos E S, Feitosa J L A, Giambroni Filho R, Azevedo J A P, Sá M P L, Bezerra A B, Jazbik A P, Murad H - Gradiente residual em operação de valva aórtica. Rev Bras Cir Cardiovasc 2000; 15(2): 136-42.

TABELA 2

\begin{tabular}{|c|c|c|c|c|c|c|}
\hline \multicolumn{7}{|c|}{ GRADIENTE FINAL SEGUNDO TAMANHO DE PRÓTESE RECOMENDADO } \\
\hline \multirow{2}{*}{$\begin{array}{l}\text { TAMANHO DE } \\
\text { PRÓTESE } \\
\text { RECOMENDADO? }\end{array}$} & \multicolumn{2}{|c|}{$<25 \mathrm{mmHg}$} & \multicolumn{2}{|c|}{$\begin{array}{c}\text { GRADIENTE FINAL } \\
\geq 25 \mathrm{~mm} H\end{array}$} & \multicolumn{2}{|c|}{ TOTAL } \\
\hline & $N$ & $\%$ & $N$ & $\%$ & $N$ & $\%$ \\
\hline Sim & 2 & 20,0 & 8 & 80,0 & 10 & 100 \\
\hline Não & 10 & 29,4 & 24 & 70,6 & 34 & 100 \\
\hline Total & 12 & 27,3 & 32 & 72,7 & 44 & 100 \\
\hline
\end{tabular}

Nota: Teste de Fisher: $p=0,702$

23 até $1,7 \mathrm{~m}^{2}$, tamanho 25 até $1,9 \mathrm{~m}^{2}$, e tamanho 27 para aqueles com superfície corpórea maior que $1,9 \mathrm{~m}^{2}$.

Aplicando à nossa população as recomendações acima citadas, encontramos apenas 10 pacientes com utilização de prótese de tamanho recomendado para sua superfície corpórea. O gradiente final médio entre eles foi $35,1 \mathrm{mmHg}$. Surpreendentemente, o gradiente final médio entre os 34 pacientes que receberam prótese de tamanho abaixo do recomendado foi $32,2 \mathrm{mmHg}(\mathrm{p}=0,553)$ (Gráfico 8$)$.

Quando tomamos o gradiente final de $25 \mathrm{mmHg}$ como referência, observamos que apenas $20 \%$ dos pacientes que receberam próteses de tamanho recomendado apresentaram gradiente final dentro deste limite, ao passo que $29,4 \%$ daqueles que receberam próteses de tamanho não recomendado apresentaram gradiente final máximo de até $25 \mathrm{mmHg}$ (Tabela 2).

\section{CONCLUSÕES}

A variação de gradiente foi diretamente proporcional ao gradiente pré-operatório.

O melhor resultado, em termos de gradiente
GRÁFICO 8

GRADIENTE FINAL SEGUNDO TAMANHODE PRÓTESE RECOMENDADO

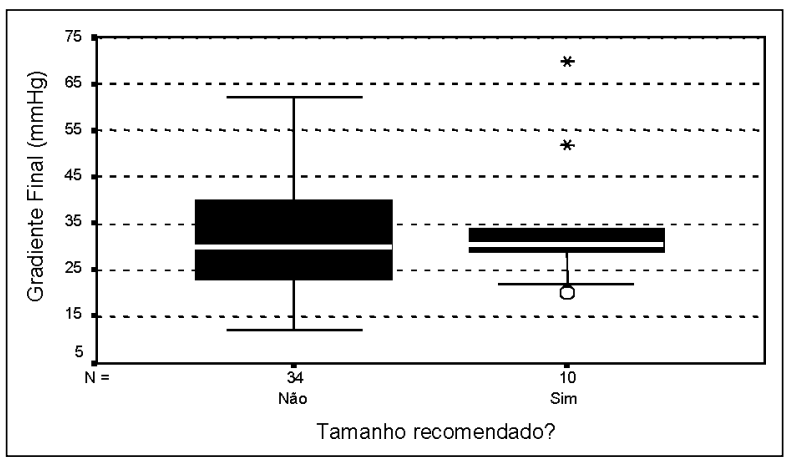

residual após operação de troca valvar aórtica, foi alcançado pelas próteses mecânicas de duplo folheto, seguidas pelas biopróteses e próteses mecânicas de monofolheto.

A utilização de próteses de tamanho menor que o recomendado, evitando o uso de procedimento de ampliação do anel aórtico, não determinou gradiente residual maior que aquele encontrado quando do implante de próteses de tamanho recomendado. 
Deucher Júnior Z, Bastos E S, Feitosa J L A, Giambroni Filho R, Azevedo J A P, Sá M P L, Bezerra A B, Jazbik A P, Murad H - Gradiente residual em operação de valva aórtica. Rev Bras Cir Cardiovasc 2000; 15(2): 136-42.

RBCCV 44205-495

Deucher Júnior Z, Bastos E S, Feitosa J L A, Giambroni Filho R, Azevedo J A R, Sá M P L, Bezerra A B, Jazbik A P, Murad H - Residual gradient in aortic valve surgery. Rev Bras Cir Cardiovasc 2000; 15(2): $136-42$.

ABSTRACT: Objectives: Evaluate residual pressure gradients after aortic valve replacement surgery in our Institution.

Material and Methods: Between January 1988 and December 1998, 44 patients with isolated aortic stenosis underwent surgery and received a valve prosthesis size 23 or smaller. Echocardiographic studies were performed at our Institution before surgery and six months postoperatively. There were $28(63.6 \%)$ males, with an average age of 53.9, and an average body surface area of $1.67 \mathrm{~m}^{2}$. Preoperative average transvalvar pressure gradient was $95.8 \mathrm{mmHg}$. Twelve patients were in functional class II, 28 in class III, and 4 in class IV. Bioprostheses were implanted in 25 patients, and mechanic prostheses in the other 19,11 of which received a bileaflet prostheses.

Results: Six months after surgery $35(79.5 \%)$ patients were in functional class I, and $9(20.5 \%)$ in class II. Average gradient variation was $62.9 \mathrm{mmHg}$. The average residual postoperative gradient was $32.9 \mathrm{mmHg}$. The gradient reduction was not influenced by sex, age, body surface area, or size of the valve prosthesis. The gradient variation was directly proportional to the preoperative gradient. Regarding type of valve prosthesis, the average residual gradient was $24.3 \mathrm{mmHg}$ for the bileaflet mechanical prosthesis, $33.7 \mathrm{mmHg}$ for the bioprosthesis, and $42.3 \mathrm{mmHg}$ for the single leaflet mechanical prosthesis.

Conclusion: Gradient variation was directly influenced by the preoperative gradient. The best results regarding residual gradients were achieved with bileaflet mechanical prostheses, followed by bioprostheses and single leaflet mechanical prostheses. Use of a smaller than recommended for body surface area prosthesis, avoiding aortic annulus enlargement procedures, was not related to higher residual gradients in this group of patients.

DESCRIPTORS: Aortic valve, surgery. Aortic valve, physiology. Aortic valve stenosis, surgery. Heart valve prosthesis, implantation.

\section{REFERÊNCIAS BIBLIOGRÁFICAS}

1 Prates $P R$ - Pequena história da cirurgia cardíaca: e tudo aconteceu diante de nossos olhos. Rev Bras Cir Cardiovas 1999; 14: 177-84.

2 De Paulis R, Sommariva L, Colagrande L et al. Regression of left ventricular hypertrophy after aortic valve replacement for aortic stenosis with different valve substitutes. J Thorac Cardiovasc Surg 1998; 116: 590-8.

3 David T E, Puschmann R, Ivanov $\mathrm{J}$ et al. - Aortic valve replacement with stentless and stented porcine valves: a case-match study. J Thorac Cardiovasc Surg 1998; 116: 236-41.

4 Bikkina M, Larson M G, Levy D - Asymptomatic ventricular arrhythmias and mortality risk in subjects with left ventricular hypertrophy. J Am Coll Cardiol 1993; 22: 1111-6.

5 Levy D, Garrison R J, Savage D D, Kannel W B, Castelli W P - Prognostic implications of echocardiographically determined left ventricular mass in the Framingham Heart Study. N Engl J Med 1990; 322: $1561-6$.

6 Arnoni R T, Arnoni A S, Meneghelo Z M et al. Evolução tardia de pacientes com prótese aórtica pequena (19 e $21 \mathrm{~mm})$. Rev Bras Cir Cardiovasc 1999; 14: 279-84.

7 Ota $\mathrm{T}$, Iwahashi $\mathrm{K}$, Matsuda $\mathrm{H}$, Tsukube $\mathrm{T}$, Ataka $\mathrm{K}$, Okada M - Reduction of left ventricular hypertrophy with St. Jude Medical $19 \mathrm{~mm}$ valve prosthesis. Angiology 1995; 46: 981-7.

8 Noera G, Pensa P, Lamarra M, Mascagni R, Cremonesi A, Balestra $G$ - Hemodynamic evaluation of the Carbomedics R, St. Jude Medical HP and Sorin-Bicarbon valve in patients with small aortic annulus. Eur J Cardiothorac Surg 1997; 11: 473-6.

9 Kirzner C F, Vinals B, Moya J et al. - Hemodynamic performance evaluation of small aortic ATS Medical valves by doppler echocardiography. J Heart Valve Dis 1997; 6: 661-5.

10 Craver J M, King S B $3^{\text {rd }}$, Douglas J S et al. - Late hemodynamic evaluation of Hancock Modified orifice aortic bioprosthesis. Circulation 1979; 60(2 Pt 2): 93-7.

11 Fiore A C, Swartz M, Grunkemeier G et al. - Valve replacement in the small aortic annulus: prospective randomized trial of St. Jude with Medtronic Hall. Eur J Cardiothorac Surg 1997; 11: 485-92.

12 Franzen S F, Huljebrant I E, Konstantinov I E, Nylan- 
Deucher Júnior Z, Bastos E S, Feitosa J L A, Giambroni Filho R, Azevedo J A P, Sá M P L, Bezerra A B, Jazbik A P, Murad H - Gradiente residual em operação de valva aórtica. Rev Bras Cir Cardiovasc 2000; 15(2): 136-42.

der $E$, Olin C L - Aortic valve replacement for aortic stenosis in patients with small aortic root. $J$ Heart Valve Dis 1996; 5(Suppl 3): S284-8.

13 Wortham D C, Tri T B, Bowen T E - Hemodynamic evaluation of the St. Jude Medical valve prosthesis in the small aortic annulus. $J$ Thorac Cardiovasc Surg 1981; 81: 615-20.

14 Sommers K E \& David T E - Aortic valve replacement with patch enlargement of the aortic annulus. Ann Thorac Surg 1997; 63: 1608-12. 